\title{
Iodine status among school age children in the Republic of Seychelles
}

\author{
A.M. Monaghan ${ }^{1}$, M.S. Mulhern ${ }^{1}$, E.M. McSorley ${ }^{1}$, J.J. Strain ${ }^{1}$, T. Winter ${ }^{2}$, E.V. Wijngaarden ${ }^{3}$, \\ G.J. Myers', C. Shamlaye ${ }^{4}$ and A.J. Yeates ${ }^{1}$ \\ ${ }^{1}$ Nutrition Innovation Centre for Food and Health, Ulster University, Coleraine, UK, \\ ${ }^{2}$ Institute of Clinical Chemistry and Laboratory Medicine, University medicine Greifswald, Greifswald, Germany, \\ ${ }^{3}$ The Department of Community and Preventive Medicine, University of Rochester School of Medicine and Dentistry, \\ Rochester, NY, USA, \\ ${ }^{4}$ The Department of Paediatrics, University of Rochester School of Medicine and Dentistry, Rochester, NY, USA and \\ ${ }^{5}$ Ministry of Health, Republic of Seychelles
}

Iodine status is associated with children's neurodevelopment. As a result, the World Health Organization (WHO) recommends the monitoring of children's iodine intakes to ensure the effective and sustainable prevention of deficiency, which is known to adversely affect child neurodevelopment ${ }^{(1,2)}$. Nevertheless, a limited amount of data on iodine status is available for developing countries, including that of the Seychelles, and as such it is imperative to evaluate child Urinary Iodine Concentrations (UIC) thus enabling monitoring of iodine status ${ }^{(3)}$. Iodine status was quantified in children from the Seychelles Child Development Study (SCDS) Nutrition Cohort 2. The Seychelles population have a high fish consumption and iodized salt is widely available4. The aim of this study was to evaluate iodine status of the Seychellois school aged children using Urinary Iodine Concentrations (UIC). We hypothesized that children in this cohort would have adequate, or even excess iodine intakes based on their dietary patterns ${ }^{(4)}$.

A total of 603 children provided a spot, non-fasted, urine samples at 7 years of age (mean 7.36 years and range 7-8 years). A $1.5 \mathrm{ml}$ aliquot was analysed at the ETH Laboratory, Zurich for UIC using the Pino modification of the standard Sandell-Kolthoff method ${ }^{(5)}$. We used the UIC data according to the WHO cut-offs for analysis5. WHO classifies I status as the following $<20 \mu \mathrm{g} / \mathrm{l} \mathrm{severe;} 20-49 \mu \mathrm{g} / 1$ moderate; $50-99 \mu \mathrm{g} / 1$ mild; $100-299 \mu \mathrm{g} / 1$ adequate and $>300 \mu \mathrm{g} / 1$ as excessive ${ }^{(6)}$.

Sufficient iodine status was observed in some $63.3 \%$ of children whereas $17.1 \%$ of children had intakes defined as mildly iodine deficient, $8.6 \%$ as moderately deficient and $2.7 \%$ as severely deficient. For $8.3 \%$ of children their UIC suggested iodine intakes were in the excessive range.

The range of UIC observed were unexpected and may reflect changing dietary patterns in the Seychelles with a shift away from high fish consumption. These findings in an oceanic, high fish-eating population warrants further research to explore the determinants of any low iodine intakes and their associations with neurodevelopmental outcomes in the children at 7 years of age.

\section{References}

1. Iodine Global Network (IGN) (2003) The WHO Global Database on iodine deficiency disorders: the importance of monitoring iodine nutrition [Available at: https://www.ign.org/p142003254.html.

2. Bath SC, Steer CD, Golding J, et al. (2013) The Lancet 382 (9889), 331-7

3. Businge CB, Longo-Mbenza B \& Kenge AP (2020) Public Health Nutr, 1-6

4. Iodine Global Network (IGN) (2020) Global scorecard of iodine nutrition in 2020. [Available at: https://www.ign.org/cm_data/ Global-Scorecard-2020-3-June-2020.pdf.

5. Pino S, Fang SL \& Braverman LE (1996) Clin Chem 42 (2), 239-243

6. World Health Organization (WHO) (2013) Urinary iodine concentrations for determining iodine status in populations [Available at: https://apps.who. int/iris/bitstream/handle/10665/85972/WHO_NMH_NHD_EPG_13.1_eng.pdf?ua=1 